\title{
Numerical solution of nonlinear the foam drainage equation via hybrid method
}

\author{
Derya Arslan \\ Department of Mathematics, Faculty of Art and Science, Bitlis Eren University, Bitlis, Turkey
}

Received: 13 September 2019, Accepted: 3 February 2020

Published online: 18 Fabruary 2020.

\begin{abstract}
In the present study, hybrid method (differential transform and finite difference methods) is selected to determine the numerical solution of the foam drainage equation. The strength of the proposed method is proven with an example for various values of $c, t$ and $x$. Numerical solutions obtained with the application of the hybrid method are compared with exact solutions. Also, in the findings section the applied method was supported with tables and figures.
\end{abstract}

Keywords: Foam drainage equation, hybrid method, differential transform method, finite difference method, approximate solution.

\section{Introduction}

Foams are quite important in certain technological processes and applications. Liquid foam is first described by Joseph Plateau. Foams are usually utilized in foods and, personal care products, acoustic cladding and textured wallpapers, etc. Also, there are important foam applications in the foods, chemical industries, fire fighting, mineral processing, and structural material sciences [1]. In nature, chemical, physical and biological events, as well as foams are often modeled with nonlinear equations. In the literature, several methods were described and implemented for the numerical solution of these models $[1,2,3,4,5,6,7,8,9,10,11,12,13,14,15]$. We, therefore, preferred a robust hybrid method to solve the following nonlinear foam drainage equation [1]:

$$
u_{t}-2 u^{2} u_{x}-\left(u_{x}\right)^{2}-\frac{1}{2} u u_{x x}=0
$$

where, the initial condition can be written as follows

$$
u(x, 0)=-\sqrt{\operatorname{ctanh}}(x \sqrt{c})
$$

and exact solution can be written as follows

$$
u(x, t)=-\sqrt{c} \tanh ((x-c t) \sqrt{c})
$$

where $c$ is defined as the velocity of the front side of the wave.

The foam drainage equation, which is a liquid flow model, is important [3]. This type of equations were discussed by various scientists. For example, soluble foam drainage model was studied in 1994 [16]. Stability of foam drainage equation was investigated in a study titled "Applications and Generalizations of Foam Drainage Equation" [17]. Physicochemical approach to foam drainage equation was studied in another study [18]. The liquid foam drainage was 
described as a nonlinear partial differential equation [19]. Partial differential equation with Caputo and Bessel operators, fractional differential equations, Riemann-Liouville fractional neutral systems, nonlinear neutral differential systems and impulsive differential equations etc. [22, 23,24,25,26,27] can be also solved by this method.

Various analytical methods were used to obtain the solution of foam drainage equation, such as Tanh method [6], spatial finite difference method, generalized $\left(G^{\prime} / G\right)$ - expansion method [5], Adomian method [6], Homotopy analysis method etc. [1]. In the literature review, it was observed that the hybrid method was never used to solve the foam drainage equation. Thus, the present study aimed to solve the foam drainage equation by the hybrid method. Hybrid method (differential transform and finite difference methods) was used by various several researchers in the fields of science and engineering $[7,8,9,10,11,12,13]$.

The intention of the present paper is to describe a novel perspective using the hybrid method for the numerical solution of nonlinear foam drainage. In Section 2, a short description of hybrid method is provided. The hybrid method was performed to a foam drainage equation example using a few steps in Section 3. The numerical findings, are presented in tables and graphs. Finally, the conclusion section completes the present study.

\section{Description of the method}

Initially, differential transform method was suggested by Zhou for linear and nonlinear equations in 1986 [20]. Chen and Ho used two-dimensional differential transform method for two-dimensional partial differential equations in 1999 [21]. Finite difference method (FDM) is an important numerical method for solving differential equations by approximation with difference equations. Thus, the hybrid procedure is obtained by applying the differential transformation method for $t$ - time variable and the finite differences method for $x$ - position variable. Based on the type problem, hybrid method provides an approximate solution in the series form or in mesh points.

Let $H(x, t)$ be the differential transformation of $h(x, t)$ function as follows [14]:

$$
h(x, t)=\sum_{k=0}^{\infty} H(i, k) t^{k}=H(i, 0)+H(i, 1) t+H(i, 2) t^{2}+\ldots
$$

The differential transform of $h(x, t)$ based on $t$-time variable is defined as follows [14]:

$$
H(i, k)=\frac{1}{k !}\left[\frac{d^{k} h(x, t)}{d t^{k}}\right]_{t=0} .
$$

The inverse of the $H(i, k)$ differential function based on $t$ is defined as follows [14].

$$
h(x, t)=\sum_{k=0}^{\infty} H(i, k) t^{k} .
$$

In Table 1 and Table 2, certain significant properties that would be used in the present study are presented [14,15].

Table 1: Properties of differential transform for $t-$ variable.

\begin{tabular}{|l|l|}
\hline Function & Transform \\
\hline$\frac{d h(x, t)}{d t}$ & $H(i, k)=(k+1) H(i, k+1)$ \\
\hline$h(x, t)=-\sqrt{c} \tanh (x \sqrt{c})$ & $H(i, k)=-\sqrt{c} \tanh \left(x_{i} \sqrt{c}\right)$ \\
\hline
\end{tabular}


Table 2: Certain properties of central difference and differential transform based on $x$ - variable.

\begin{tabular}{|l|l|}
\hline Function & Transform \\
\hline$\frac{\partial w(x, t)}{\partial x}$ & $W(i, k)=\frac{W(i+1, k)-W(i-1, k)}{2 h}$ \\
\hline$\frac{\partial^{2} w(x, t)}{\partial x^{2}}$ & $\frac{W(i+1, k)-2 W(i, k)+W(i-1, k)}{h^{2}}$ \\
\hline$\left(\frac{\partial w(x, t)}{\partial x}\right)^{2}$ & $\sum_{m=0}^{k}\left(\frac{W(i+1, k-m)-W(i-1, k-m)}{4 h^{2}}\right)(W(i+1, k)-W(i-1, k))$ \\
\hline$w(x, t) \frac{\partial^{2} w(x, t)}{\partial x^{2}}$ & $\sum_{m=0}^{k} W(i, k-m) \frac{W(i+1, k)-2 W(i, k)+W(i-1, k)}{h^{2}}$ \\
\hline$w^{2}(x, t) \frac{\partial w(x, t)}{\partial x}$ & $\sum_{m=0}^{k} \sum_{t=0}^{k-m} \frac{1}{2 h} W(i, m) W(i, t)(W(i+1, k-m-t)-W(i-1, k-m-t))$ \\
\hline $\tanh (x \sqrt{c})$ & $\tanh \left(x_{i} \sqrt{c}\right)$ \\
\hline
\end{tabular}

\section{The comparison of exact and approximation solutions with the Hybrid method}

Here, an example will be given to demonstrate the applicability of the adopted method. All calculations and plots are conducted with a computer software. The findings are cross-checked.

\section{Example 1.}

The foam drainage equation with the initial condition is considered as an illustrative example [1],

$$
u_{t}-2 u^{2} u_{x}-\left(u_{x}\right)^{2}-\frac{1}{2} u u_{x x}=0
$$

and the initial condition and exact solution are given below:

$$
\begin{gathered}
u(x, 0)=-\sqrt{c} \tanh (x \sqrt{c}), \\
u(x, t)=-\sqrt{c} \tanh ((x-c t) \sqrt{c}) .
\end{gathered}
$$

Computational procedure based on the hybrid method is given below:

Based on the Table 1 and Table 2, we can demonstrate the recurrence relation from (6)-(7) as follows:

$$
\begin{aligned}
U(k+1) & =\frac{1}{(k+1)}\left(-2 \sum_{m=0}^{k}\left(\sum_{t=0}^{k-m} \frac{1}{2 h} U(i, m) U(i, t)(U(i+1, k-m-t)-U(i-1, k-m-t))\right)\right) \\
& +\sum_{m=0}^{k}(U(i+1, k)-U(i-1, k))\left(\frac{U(i+1, k-m)-U(i-1, k-m)}{4 h^{2}}\right) \\
& +\sum_{m=0}^{k} U(i, k-m)\left(\frac{U(i+1, k)-2 U(i, k)+U(i-1, k)}{2 h^{2}}\right), k=0,1,2, \ldots
\end{aligned}
$$

After the differential transform coefficients $U(i, 0), U(i, 1), U(i, 2) \ldots$ are obtained with recurrence relation for $k=1,2,3, \ldots$, the following approximate solutions are obtained using (5) for $t=0.001, c=1$ and presented in Table 1 :

$$
\begin{aligned}
x_{i}=0, u(0, t) & =\sum_{k=0}^{\infty} U(0, k) t^{k}=U(0,0)+U(0,1) t+U(0,2) t^{2}+\ldots \\
& =-0.5800256585 t-0.9293491752 t^{2}-0.9901339629 t^{3}-\ldots-0.9999999918 t^{10} .
\end{aligned}
$$




$$
\begin{aligned}
x_{i}=0.1, u(0.1, t) & =\sum_{k=0}^{\infty} U(0.1, k) t^{k}=U(0.1,0)+U(0.1,1) t+U(0.1,2) t^{2}+\ldots \\
& =-0.009933709152-0.5130826389 t-0.9143900763 t^{2}-\ldots-0.9999999900 t^{10} .
\end{aligned}
$$

The same calculations are performed at the other points $x_{i}$ in a similar way.

$$
\begin{aligned}
x_{i}=1, u(1, t) & =\sum_{k=0}^{\infty} U(1, k) t^{k}=U(1,0)+U(1,1) t+U(1,2) t^{2}+\ldots \\
& =-0.5800256585-0.5800256585 t^{2}-0.9293491752 t^{3}-\ldots-0.9999999390 t^{10}
\end{aligned}
$$

where $x_{i}=i h$ mesh points for $h=0.1, i=0,1,2, \ldots, 10$. The following approximate solutions are obtained with (5) for $t=0.0001, c=2$ and presented in Table 2:

$$
\begin{aligned}
x_{i}=0, u(0, t) & =\sum_{k=0}^{\infty} U(0, k) t^{k}=U(0,0)+U(0,1) t+U(0,2) t^{2}+\ldots \\
& =-1.394588760 t-1.414144525 t^{2}-1.414213321 t^{3}-\ldots-1.414213562 t^{10} \\
x_{i}=0.1, u(0.1, t) & =\sum_{k=0}^{\infty} U(0.1, k) t^{k}=U(0.1,0)+U(0.1,1) t+U(0.1,2) t^{2}+\ldots \\
& =-0.02791137759-1.388232650 t-1.414121957 t^{2}-\ldots-1.414213562 t^{10} .
\end{aligned}
$$

The same calculations are obtained in the other points $x_{i}$ in a similar manner.

$$
\begin{aligned}
x_{i}=1, u(1, t) & =\sum_{k=0}^{\infty} U(1, k) t^{k}=U(1,0)+U(1,1) t+U(1,2) t^{2}+\ldots \\
& =-1.116138222-1.116138222 t-1.413045987 t^{2}-\ldots-1.414213562 t^{10}
\end{aligned}
$$

The following approximate solutions are found with (5) for $t=0.00001, c=2$ and presented in Table 3:

$$
\begin{gathered}
x_{i}=0, \begin{array}{c}
u(0, t)=\sum_{k=0}^{\infty} U(0, k) t^{k}=U(0,0)+U(0,1) t+U(0,2) t^{2}+\ldots \\
=-1.394588760 t-1.414144525 t^{2}-\ldots-1.414213562 t^{10} . \\
x_{i}=0.1, u(0.1, t)=\sum_{k=0}^{\infty} U(0.1, k) t^{k}=U(0.1,0)+U(0.1,1) t+U(0.1,2) t^{2}+\ldots \\
=-0.02791137759-1.388232650 t-1.414121957 t^{2}-\ldots-1.414213562 t^{10} .
\end{array}
\end{gathered}
$$

The same calculations are obtained in the other points $x_{i}$ in a similar manner.

$$
\begin{aligned}
x_{i}=1, u(1, t) & =\sum_{k=0}^{\infty} U(1, k) t^{k}=U(1,0)+U(1,1) t+U(1,2) t^{2}+\ldots \\
& =-1.116138222-1.116138222 t-1.413045987 t^{2}-\ldots-1.414213562 t^{10}
\end{aligned}
$$

It could be observed that the approximate solutions obtained with the hybrid method and exact solutions are quite similar. 

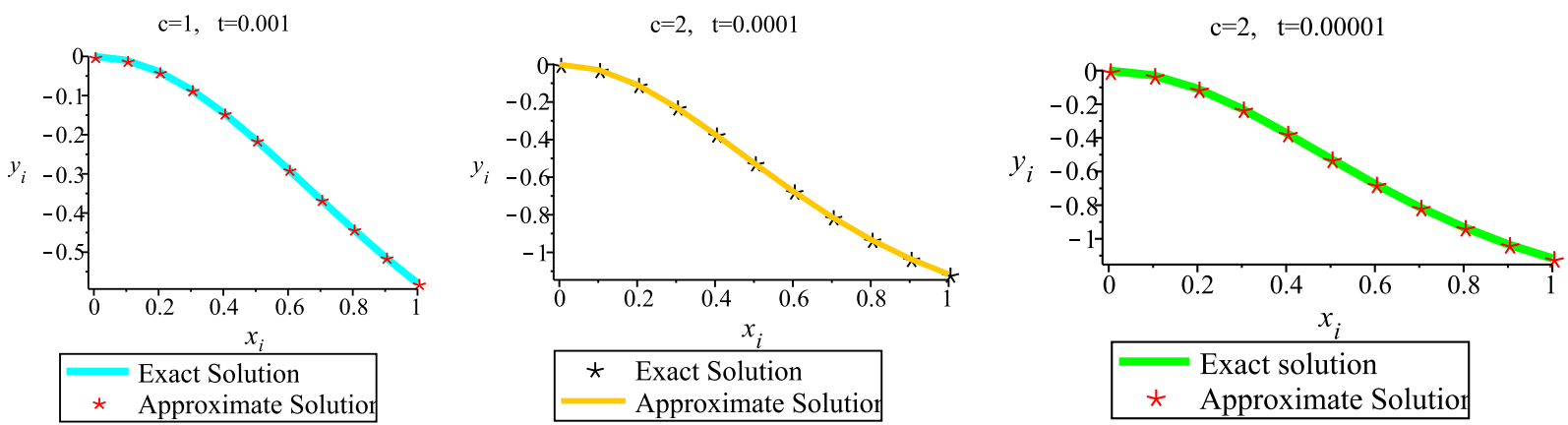

Fig. 1: The curves obtained with the hybrid method and exact solution for different $t$ and $c$ values.

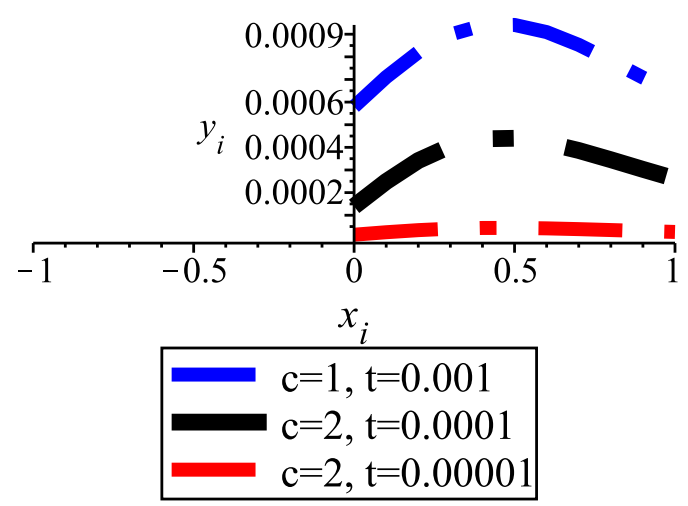

Fig. 2: The comparison of errors for different $t$ and $c$ values.

The exact, approximate solutions and error values for different $t$ and $c$ values are presented in the following tables:

Table 3: The approximate solution and, the exact solution and the error values for $c=1, t=0.001$.

\begin{tabular}{|l|l|l|l|}
\hline$x\left(x_{i}\right)$ & Hybrid method & Exact & Error of hybrid method \\
\hline 0.0 & $-0.5809559988 e-3$ & $-0.9999993334 e-6$ & $0.5799559995 e-3$ \\
\hline 0.2 & $-0.3939885928 e-1$ & $-0.3857849389 e-1$ & $0.82036539 e-3$ \\
\hline 0.4 & -0.1446504867 & -0.1437115015 & $0.9389852 e-3$ \\
\hline 0.6 & -0.2885673834 & -0.2876580288 & $0.9093546 e-3$ \\
\hline 0.8 & -0.4409844853 & -0.4402021857 & $0.7822996 e-3$ \\
\hline 1.0 & -0.5800262394 & -0.5793856477 & $0.6405917 e-3$ \\
\hline
\end{tabular}

Foam drainage equation is solved numerically using the hybrid method. Concurrently, the given hybrid method solution provided a quick convergence as presented in Table 3, Table 4 and Table 5. Also, the approximate and the exact solutions are compared. These solutions are presented in Figure 1 and Figure 2, and indicated that the presented method is effective and powerful. 
Table 4: The approximate solution and, the exact solution and the error values for $c=2, t=0.0001$.

\begin{tabular}{|l|l|l|l|}
\hline$x\left(x_{i}\right)$ & Hybrid method & Exact & Error of Hybrid method \\
\hline 0.0 & $-0.1394730188 e-3$ & $-0.1131370789 e-6$ & $0.1393598817 e-3$ \\
\hline 0.2 & -0.1075036796 & -0.1071620693 & $0.3416103 e-3$ \\
\hline 0.4 & -0.3711289566 & -0.3706912773 & $0.4376793 e-3$ \\
\hline 0.6 & -0.6740232547 & -0.6736030899 & $0.4201648 e-3$ \\
\hline 0.8 & -0.9314011508 & -0.9310557636 & $0.3453872 e-3$ \\
\hline 1.0 & -1.116249850 & -1.115988393 & $0.261457 e-3$ \\
\hline
\end{tabular}

Table 5: The approximate solution and, the exact solution and the error values for $c=2, t=0.00001$.

\begin{tabular}{|l|l|l|l|}
\hline$x\left(x_{i}\right)$ & Hybrid method & Exact & Error of Hybrid method \\
\hline 0.0 & $-0.1394602901 e-4$ & $-0.1131370848 e-8$ & $0.1394489764 e-4$ \\
\hline 0.2 & -0.1073794797 & -0.1073453127 & $0.341670 e-4$ \\
\hline 0.4 & -0.3710070594 & -0.3709632911 & $0.437683 e-4$ \\
\hline 0.6 & -0.6739053089 & -0.6738632961 & $0.420128 e-4$ \\
\hline 0.8 & -0.9312898566 & -0.9312553219 & $0.345347 e-4$ \\
\hline 1.0 & -1.116149383 & -1.116123242 & $0.26141 e-4$ \\
\hline
\end{tabular}

\section{Conclusion}

In particular, literature review demonstrated that it is hard to solve most nonlinear differential equations exactly. Thus, approximate and numerical methods are used extensively. With the numerical hybrid method, we could successfully obtain numerical solutions in the present study for different values of $c, t$ and $x$. These solutions are presented in tables and figures, revealing that the solutions were accurate and proposed methods were strong and efficient. In conclusion, it was revealed these methods were as good as the other methods based on fast convergence. In brief, the proposed methods were potential significant approximation methods that could be used in further studies on nonlinear partial differential equations.

\section{Acknowledgement}

The author is grateful to the anonymous reviewers for his suggestions and comments for helping to augment the quality of the manuscript.

\section{Competing interests}

The authors declare that they have no competing interests.

\section{Authors' contributions}

All authors have contributed to all parts of the article. All authors read and approved the final manuscript.

\section{References}

[1] Sadigh Behzadi, Sh., The use of homotopy methods for solving nonlinear foam drainage equation, Communications on Advanced Computational Science with Applications, 2014: 1-18, (2014). 
[2] Darvishi, M.T. and Khani, F., A series solution of the foam drainage equation, Computers and Mathematics with Applications, 58: 360-368, (2009).

[3] Dahmani, Z., Mesmoudi, M.M. and Bebbouchi, R., The foam drainage equation with time- and space-fractional derivatives solved by the Adomian method, Electronic Journal of Qualitative Theory of Differential Equations, 2008(30): 1-10, (2008).

[4] Cilingir Sungu, I. and Demir, H., Temporal differential transform and spatial finite difference methods for unsteady heat conduction equations with anisotropic diffusivity, Gazi University Journal of Science, 27(4): 1063-1076, (2014).

[5] Nur Alam, Md., Exact solutions to the foam drainage equation by using the new generalized $\left(G^{\prime} / G\right)$ - expansion method, Results in Physics, 5: 168-177, (2015).

[6] Helal, M.A. and Mehanna, M.S., The tanh method and Adomian decomposition method for solving the foam drainage equation, Applied Mathematics and Computation, 190(2007): 599-609, (2007).

[7] Chen, C.K., Lai, H.Y. and Liu, C.C., Nonlinear micro circular plate analysis using hybrid differential transformation/finite difference method, CMES 40, 155-174, (2009).

[8] Chu, S.P., Hybrid differential transform and finite difference method to solve the nonlinear heat conduction problems, WHAMPOA- An Interdisciplinary Journal, 66: 15-26, (2014).

[9] Chu, H.P. and Chen, C.I., Hybrid differential transform and finite difference method to solve the nonlinear heat conduction problem, Communication in Nonlinear Science and Numerical Simulation, 13: 1605-1614, (2008).

[10] Sungu, I. and Demir, H., Solutions of the system of differential equations by differential transform/finite difference method, NwsaPhysical Sciences, 7: 1308-7304, (2012).

[11] Sungu, I. and Demir, H., A new approach and solution technique to solve time fractional nonlinear reaction-diffusion equations, Hindawi Publishing Corporation Mathematical Problems in Engineering, 13 pages, (2015).

[12] Sungu, I. and Demir, H., Application of the hybrid differential transform method to the nonlinear equations, Applied Mathematics, 3: 246-250, (2012).

[13] Yeh, Y.L., Wang, C.C. and Jang, M.J., Using finite difference and differential transformation method to analyze of large deflections of orthotropic rectangular plate problem, Applied Mathematics and Computation, 190(2007): 1146-1156, (2007).

[14] Ayaz, F., On the two-dimensional differential transform method, Applied Mathematics and Computation, 1432(2-3): 361-374, (2003).

[15] Cakir M. and Arslan, D., The Adomian decomposition method and the differential transform method for numerical solution of multi Pantograph delay differential equations, Applied Mathematics, 6:1332-1343, (2015).

[16] Verbist, G. and Weaire, D., Soluble model for foam drainage, Europhysics Letters, 26: 631-641, (1994).

[17] Cox, S.J., Weaire, D., Hutzler, S., Murphy, J., Phelan, R. and Verbist, G., Applications and generalizations of the foam drainage equation, Proceedings of the Royal Society of London, Series A: Mathematical, Physical and Engineering, 456: 2441-2464, (2000).

[18] Durand, M. and Langevin, D., Physicochemical approach to the theory of foam drainage, The European Physical Journal E, 7(1): 35-44, (2002).

[19] Verbist, G., Weaire, D. and Kranyik, A.M., The foam drainage equation, Journal of Physics: Condensed Matter, 8(21): 3715-3731, (1996).

[20] Zhou, J.K., Differential transformation and its applications for electrical circuits, Huazhong University Press, Wuhan, China, (1986).

[21] Chen, C.K. and Ho, S.H., Solving partial differential equations by two dimensional differential transform method, Applied Mathematics and Computation, 106: 171-179, (1999).

[22] Agarwal, P., Al-Mdallal, Q., Je Cho, Y. and Jain, S., Fractional differential equations for the generalized Mittag-Leffler function, Advances in Difference Equations 2018(1), 58: (2018).

[23] Zhang, X., Agarwal, P., Liu, Z. and Peng, H., The general solution for impulsive differential equations with Riemann-Liouville fractional-order $q \in(1,2)$, Open Mathematics 13(1): (2015).

[24] Agarwal, P., Karimov, E., Mamchuev, M. and Ruzhansky, M., On boundary-value problems for a partial differential equation with Caputo and Bessel operators, Birkhauser, Cham, Book, (2016).

[25] Pesenson, I., Le Gia, Q.T., Mayeli, A., Mhaskar, H. and Zhou, D., Recent applications of harmonic analysis to function spaces, differential equations, and data science, 707-718, (2018).

[26] Altun, Y., Further results on the asymptotic stability of Riemann-Liouville fractional neutral systems with variable delays, Advances in Difference Equations, 2019(1): 437, (2019). https://doi.org/10.1186/s13662-019-2366-z. 
[27] Altun, Y., On exponential stability of solutions of nonlinear neutral differential systems with discrete and distributed variable lags, Nonlinear Studies, 26(2): (2019). 